\title{
CONTRIBUTION OF THE RECENT AUSM SCHEMES TO THE OVERFLOW CODE: IMPLEMENTATION AND VALIDATION
}

\author{
Meng-Sing Liou* \\ NASA Glenn Research Center, MS 5-11, Cleveland, OH 44135, USA \\ (216) 433-58.55, fsmsl@yinyan grc.nasa.gov \\ and \\ Pieter G. Buning ${ }^{\dagger}$ \\ NASA Langley Research Center, MS 499, Hampton, VA 23681, USA \\ (757) 864-3093, p.g.buning@larc.nasa.gov
}

\begin{abstract}
We shall present results of a recent collaborative effort between the authors attempting to implement the numerical flux scheme, AUSM ${ }^{+}$and its new developments, into a widely used NASA code, OVERFLOW. This paper is intended to give a thorough and systematic documentation about the solutions of default test cases using the $\mathrm{AUSM}^{+}$scheme. Hence we will address various aspects of numerical solutions, such as accuracy, convergence rate, and effects of turbulence models, over a variety of geometries, speed regimes.

We will briefly describe the numerical schemes employed in the calculations, including the capability of solving for low-speed flows and multiphase flows by employing the concept of numerical speed of sound. As a bonus, this low Mach number formulations also enhances convergence to steady solutions for flows even at transonic speed. Calculations for complex 3D turbulent flows were performed with several turbulence models and the results display excellent agreements with measured data.
\end{abstract}

\section{INTRODUCTION}

A collaborative attempt has been evolved to implement the numerical flux scheme, $\mathrm{AUSM}^{+}$and its new developments [1-5], into a NASA CFD code, OVERFLOW [6]. The OVERFLOW code currently is a production code that has been widely used in the US industry, government labs,

\footnotetext{
*Associate Fellow, AIAA

$\dagger$ Associate Fellow, AlAA

This paper is declared a work of the U.S. Government and is not subject to copying right protection in the United States.
}

and universities. It is written to use the overset, structured (Chimera) grid systems and has evolved through several significant changes, due to contributions from various researchers over more than two decades (see [6] for further details and references therein). The Chimera grid approach was developed for multibodies and complex geometry flow simulations. Major applications have included the Space Shuttle Launch Vehicle and subsonic transport high-lift configurations. The Pulliam-Chaussee diagonalized implicit algorithm [16] has become the standard solver for the code. Several numerical flux schemes are commonly used, including central differencing and Roe upwind differencing.

The AUSM family schemes, even though relatively young, also has gone through several modifications. The schemes have been made into some major production codes as well (see, for example, [7]-[9]).

In addition, this collaboration is intended to give a thorough and systematic documentation about the solutions of default test cases obtained by using the newly implemented scheme. Hence, it is considered to be a long-term project, especially the validation processes. As a result, this paper contains only the first installment of results from this continuing effort. The numerical schemes will be briefly described, together with the concept of numerical speed of sound [5], which is especially useful for low speed flows and multiphase flows $[11,17]$. To confirm the effectiveness 
of the $\mathrm{AUSM}^{+}$scheme for practical engineering problems, calculations for a wide variety of flows were made. The results display excellent agreements with measured data.

\section{NUMERICAL METHODS}

\section{Governing Equations}

We solve the Reynolds-averaged Navier-Stokes equations written in curvilinear coordinates $(\xi, \eta, \zeta)$ and conservation form:

$$
\mathbf{Q}_{t}+\mathbf{F}_{l, l}+\mathbf{F}_{l, l}^{(\mathrm{v})}=0, \quad l=1,2,3(\xi, \eta, \zeta),
$$

where

$$
\mathbf{Q}=\left(\rho, \rho u, \rho v, \rho w, \rho \epsilon_{t}\right)^{T} / J .
$$

The inviscid and viscous fluxes are included in $\mathbf{F}_{l, l}$ and $\mathbf{F}_{l, l}^{(\mathbf{v})}$, where the summation and differentiation notation, $\mathbf{F}_{l, l}=\sum_{l=\mathbf{1}}^{3} \partial \mathbf{F}_{l} / \partial x_{l}$ is assumed.

$\mathbf{F}_{l}=\left(\begin{array}{c}\rho u_{l} \\ \rho u_{l} u_{1}+p \delta_{l 1} \\ \rho u_{l} u_{2}+p \delta_{l 2} \\ \rho u_{l} u_{3}+p \delta_{l 3} \\ \rho u_{l} h_{t}\end{array}\right), \quad \mathbf{F}_{l}^{(v)}=-\left(\begin{array}{c}0 \\ \tau_{1 l} \\ \tau_{2 l} \\ \tau_{3 l} \\ \kappa T_{, l}+u_{i} \tau_{i l}\end{array}\right)$

where

$$
\tau_{i j}=\mu\left(u_{i, j}+u_{j, i}-\frac{2}{3} u_{i, i}\right)
$$

The flow variables in $\mathbf{Q}$ and the geometric Jacobian $J$ are standard. The turbulence terms are included in $\mathbf{F}^{(\mathbf{v})}$ and expressed in the form of eddy-viscosity described by algebraic or differential equations.

To enhance the convergence rate when solving flows at low speeds, the time-derivative term is premultiplied by a conditioning matrix $\boldsymbol{\Gamma}$. The equations now can be cast as,

$$
\Gamma \tilde{\mathbf{Q}}_{t}+\mathbf{F}_{l, l}+\mathbf{F}_{l, l}^{(\mathrm{v})}=0, \quad \tilde{\mathbf{Q}}=(p, u, v, w, T)^{T} / J
$$

Several forms for the local preconditioning matrix $\Gamma$ have been proposed, notably by Choi and Merkle [12], Turkel [13], and Van Leer, Lee, and Roe [14]. While these matrices are different, owing to different design criteria, it is not essential for now to explicitly specify the form of $\Gamma$.

\section{Implicit Operator}

The nonlinear equations of (4) are linearized, resulting in an implicit equation to solve for $\Delta \mathbf{Q}=\mathbf{Q}^{\mathbf{n + 1}}-\mathbf{Q}^{\mathbf{n}}$.

$$
\begin{aligned}
& {\left[\mathbf{I}+\Delta t \delta_{l}\left(\mathbf{M} \Gamma^{-1} \mathbf{A}_{l}\right)\right] \Delta \mathbf{Q}} \\
& =-\Delta t \mathbf{M} \Gamma^{-1} \delta_{l}\left(\mathbf{F}_{l}+\mathbf{F}_{l}^{(\mathbf{v})}\right)
\end{aligned}
$$

where

$$
\mathbf{M}=\frac{\partial \mathbf{Q}}{\partial \tilde{\mathbf{Q}}}, \quad \mathbf{A}_{l}=\frac{\partial \mathbf{F}_{l}}{\partial \mathbf{Q}} .
$$

Note that the LHS implicit operator consists of only the inviscid part of the whole flux. Several methods have been suggested for approximating the implicit operator, in order to gain convergence rate, reduce computation, and increase stability. Several options are available in the OVERFLOW code. At the initial stage of this study, our focus is mostly on the accuracy and convergence rate of steady solutions, which are controlled only by the numerical methods representing the RHS and should be independent of the LHS operator because it vanishes as steady solutions are reached. In this study, we fixed the implicit operator to be the Beam-Warming ADI scheme [15], in which the LHS was approximately factored according to the computation coordinates in the following form:

$$
\begin{gathered}
{\left[\mathbf{I}+\Delta t \delta_{1} \mathbf{N} \mathbf{A}_{1}\right]\left[\mathbf{I}+\Delta t \delta_{2} \mathbf{N} \mathbf{A}_{2}\right]\left[\mathbf{I}+\Delta t \delta_{3} \mathbf{N} \mathbf{A}_{3}\right] \Delta \mathbf{Q}} \\
=-\Delta t \mathbf{N} \delta_{l}\left(\mathbf{F}_{l}+\mathbf{F}_{l}^{(\mathbf{v})}\right), \quad \mathbf{N}=\mathbf{M} \Gamma^{-1} .
\end{gathered}
$$

The LHS operator was then discretized using the central differencing method, with appropriate second-order and fourth-order smoothing terms added for stability. Furthermore, the diagonalization procedure of Pulliam-Chaussee [16] was applied. Details can be found in [6]. Since the RIIS operator was represented using the $\mathrm{AUSM}^{+}$scheme - an upwind scheme, there should be a concern about the inconsistency in discretization of the LUS and RHS terms. And the stability and convergence rate are likely not at optimal situations. This, however, is a very 
difficult issue by itself and will not be addressed in the present paper.

\section{Explicit Operator}

In this section, we will give a brief description of the algorithm involved in the $\mathrm{AUSM}^{+}[2]$ and the new development for the low-speed flows $[5,11,17]$. Concerning detailed numerical properties and detailed analysis, the reader should consult the cited references.

For illustration purposes, we shall begin by considering the 1D flux for ideal gas. The inviscid flux $\mathbf{F}$ is written as a sum of convective and pressure fluxes:

$\mathbf{F}=\rho u\left(\begin{array}{c}1 \\ u \\ H\end{array}\right)+\left(\begin{array}{l}0 \\ p \\ 0\end{array}\right)=\dot{m}\left(\begin{array}{c}1 \\ u \\ H\end{array}\right)+\left(\begin{array}{l}0 \\ p \\ 0\end{array}\right)=\dot{m} \mathbf{\Phi}+\mathbf{p}$.

The vectors $\boldsymbol{\Phi}$ and $\mathbf{p}$ are introduced respectively as shown. It is noted that a common mass flux $\dot{m}$ appears in all equations. This is also true for multidimensions. Since the mass flux is common for all equations, its effects will thus perpetuate in all variables. Hence, it is desirable observing this fact at the discrete level when devising a new scheme. However, this fact is not entirely enforced in several modern numerical schemes. If this same factor is kept at the discrete level for all equations, it becomes easy to add other conservation laws.

As observed in (8), all one needs to do is to define the quantities, $\dot{m}, \boldsymbol{\Phi}$ and $\mathbf{p}$. Concerning the details of developments of the AUSM-family fluxes, the interested readers are referred to the references $[1-5,11]$. In what follows we will summarize only the latest formulas implemented in the code [5]. Firstly, a simple upwinding is applied to the convected quantity $\Phi$ to yield the numerical flux at the cell interface (denoted by subscript 1/2) straddling cells $j$ and $j+1$.

$\mathbf{f}_{1 / 2}=\frac{1}{2}\left[\dot{m}\left(\boldsymbol{\Phi}_{j}+\boldsymbol{\Phi}_{j+1}\right)+|\dot{m}|\left(\boldsymbol{\Phi}_{j}-\boldsymbol{\Phi}_{j+1}\right)\right]+\mathbf{p}_{1 / 2}$.

The pressure flux is nothing but

$$
p_{1 / 2}=\mathcal{P}_{(5, \alpha)}^{+}\left(M_{j}\right) p_{j}+\mathcal{P}_{(5, \alpha)}^{-}\left(M_{j+1}\right) p_{j+1},
$$

with

$$
\mathcal{P}_{(5, \alpha)}^{ \pm}(M)=\left\{\begin{array}{c}
\frac{1}{2 M}(M \pm|M|), \quad \text { if }|M| \geq 1, \\
\frac{1}{4}(M \pm 1)^{2}(2 \mp M) \\
\pm \frac{3}{16} M\left(M^{2}-1\right)^{2} ; \text { otherwise. }
\end{array}\right.
$$

The only task left now is to find the mass flux $\dot{m}$, which can be easily done by following these steps.

1. A concept of numerical speed of sound is introduced in [5] to make the flux formulation valid for the entire speed regime. Here, we let the speed of sound be scaled by a scaling factor $f_{a}\left(\bar{M} ; M_{*}\right)$,

$$
\tilde{a}_{1 / 2}=f_{a}\left(\bar{M} ; M_{*}\right) a_{1 / 2},
$$

where

$$
\begin{aligned}
f_{a}\left(M ; M_{*}\right) & =\frac{\sqrt{\left(1-M_{*}^{2}\right)^{2} M^{2}+4 M_{*}^{2}}}{1+M_{*}^{2}} \leq 1, \\
M & =\left(M_{j}+M_{j+1}\right) / 2,
\end{aligned}
$$

and

$$
M_{*}^{2}=\min \left(1, \max \left(M^{2}, M_{c o}^{2}\right)\right)
$$

The unscaled speed of sound $a_{1 / 2}$ can take several forms, as given in $[2,4]$, one of which allows exact capturing of stationary shock. For simplicity, a simple average suffices,

$$
a_{1 / 2}=\left(a_{j}+a_{j+1}\right) / 2 .
$$

The cutoff parameter $M_{c o}<<1$ is introduced to prevent a singularity at stagnation point. It is a user-specified parameter and we choose $M_{c o}^{2}=M_{\infty}^{2} / 4$. The use of numerical speed of sound $\tilde{a}$ results in a reduction in numerical dissipation at low Mach numbers.

2. Use $\tilde{a}$ to define

$$
\tilde{M}_{j / j+1}=\frac{u_{j / j+1}}{\tilde{a}_{1 / 2}}=\frac{M_{j / j+1}}{f_{a}} .
$$


This scaled Mach number will revert to the local physical Mach number at supersonic speeds. In fact, the scaling factor becomes essential only in the low Mach range since it rises rather quickly towards unity as $M$ increases. Hence, what the numerical speed of sound does is to make the Mach number appear larger in determining the numerical fluxes in the low speed regime.

3. Let

$$
\begin{gathered}
\hat{M}_{j}=\frac{1}{2}\left[\left(1+M_{*}^{2}\right) \tilde{M}_{j}+\left(1-M_{*}^{2}\right) \tilde{M}_{j+1}\right], \\
\hat{M}_{j+1}=\frac{1}{2}\left[\left(1+M_{*}^{2}\right) \tilde{M}_{j+1}+\left(1-M_{*}^{2}\right) \tilde{M}_{j}\right] .
\end{gathered}
$$

4. Set the interface Mach number,

$$
M_{1 / 2}=\mathcal{M}_{(4, \beta)}^{+}\left(\hat{M}_{j}\right)+\mathcal{M}_{(4, \beta)}^{-}\left(\hat{M}_{j+1}\right) .
$$

The split Mach number functions, $\mathcal{M}_{(4, \beta)}^{ \pm}$, are defined as follows:

$$
\mathcal{M}_{(4, \beta)}^{ \pm}(M)=\left\{\begin{array}{c}
\frac{1}{2}(M \pm|M|), \quad \text { if }|M| \geq 1 \\
\pm \frac{1}{4}(M \pm 1)^{2} \pm \frac{1}{8}\left(M^{2}-1\right)^{2} \\
\text { otherwise. }
\end{array}\right.
$$

The numeral in the subscript of $\mathcal{M}_{(4,3)}^{ \pm} \dagger$ indicates the degree of polynomials used.

5. The mass flux can now be obtained by a simple upwind formula,

$$
\begin{aligned}
\dot{m}_{1 / 2}= & \frac{\tilde{a}_{1 / 2}}{2}\left[M_{1 / 2}\left(\rho_{j}+\rho_{j+1}\right)\right. \\
& \left.+\left|M_{1 / 2}\right|\left(\rho_{j}-\rho_{j+1}\right)\right],
\end{aligned}
$$

For identification purpose, we now call this extended method, $\mathrm{AUSM}^{+}-$a, to highlight the role of the numerical speed of sound $\tilde{a}$.

Remark: Test cases have shown that the definitions in Eqs. (18)-(19) are not strictly necessary, the simpler alternatives $\hat{M}_{j}=\dot{M}_{j}$ and $\hat{M}_{j+1}=\tilde{M}_{j+1}$ can be used as well.

To demonstrate the robustness, shock-capturing capability, and positivity-preserving property all at once, the moving shock around a 90-degree corner is a suitable problem. Several modern upwind methods have been known to encounter difficulties [4]. Figure 1 displays the density contours, showing crisp resolution of the original and several internal shocks, as well as the shear layers resulting from the intersection of shocks.

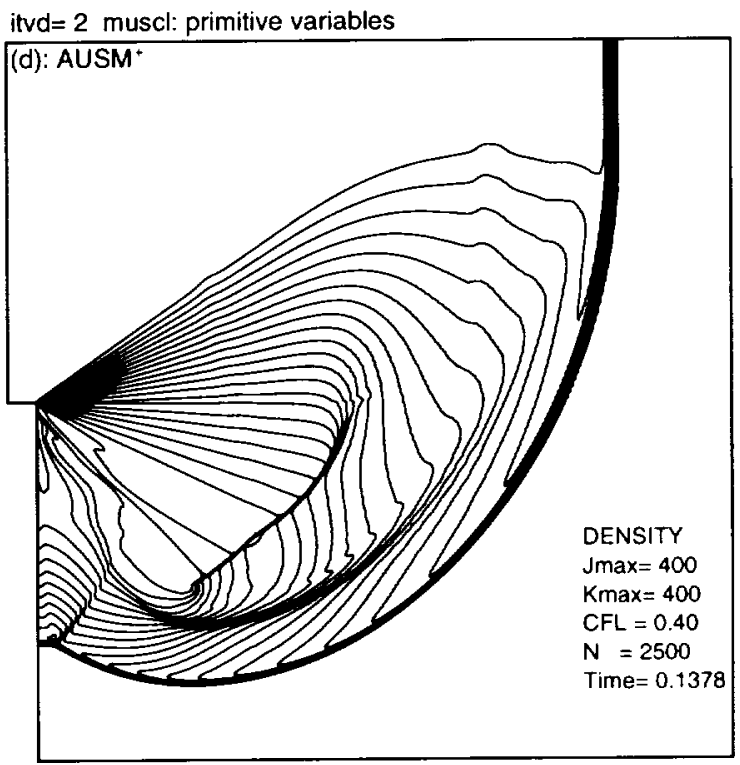

Fig. 1 Supersonic corner problem. Calculation was made with linear extrapolation of primitive variables using the Van Albada limiter

To see the effect of this numerical speed of sound, we evaluate the condition number at low speed,

$$
\kappa=\frac{|u|+\tilde{a}}{|u|} \rightarrow O(1),
$$

because

$$
\tilde{a} \rightarrow 0, \quad \text { as }|u| \rightarrow 0 .
$$

Hence, the condition number remains of order unity for all speed ranges. Also, the numerical dissipation based on this new speed of sound now scales with local speed $|u|$, instead of local speed of sound $a$ as $|u| \rightarrow 0$.

t The coefficient in (19b) of JCP129, 364-382 (1996), should be $1 / 4$, not $1 / 2$. 
To further improve the residual convergence in the low speed range, it is found beneficial in Edwards and Liou [11] if a pressure diffusion term $\dot{m}_{p}$ is included in the mass flux.

$$
\dot{m}_{1 / 2}=\text { Eq. }(22)+\dot{m}_{p} .
$$

Here $\dot{m}_{p}$ is expressed in a general form,

$$
\dot{m}_{p}=\frac{\tilde{a}_{1 / 2}}{\mathcal{D}}\left(1-M_{*}^{2}\right) \Delta \mathcal{M}\left(p_{j}-p_{j+1}\right)
$$

where

$$
\begin{aligned}
& \Delta \mathcal{M}=\left[\mathcal{M}_{(4, \beta)}^{+}\left(\hat{M}_{j}\right)-\mathcal{M}_{(1)}^{+}\left(\hat{M}_{j}\right)\right. \\
& \left.-\mathcal{M}_{(4, \beta)}^{-}\left(\hat{M}_{j+1}\right)+\mathcal{M}_{(1)}^{-}\left(\hat{M}_{j+1}\right)\right]
\end{aligned}
$$

and the function $\mathcal{D}$ in the denominator can take several forms. Based on the mass flux of the AUSMDV scheme, Edwards and Liou [11] derived the pressure diffusion term for low Mach numbers,

$$
\mathcal{D}=M_{*}^{2}\left[\frac{p_{j}}{\rho_{j}}+\frac{p_{j+1}}{\rho_{j+1}}\right] .
$$

Another formula has been proposed [5],

$$
\begin{array}{r}
\mathcal{D}=\frac{1}{\rho_{j} \rho_{j+1}}\left[M_{*}^{2}\left(p_{j}+p_{j+1}\right)\left(\rho_{j}+\rho_{j+1}\right)\right. \\
\left.-\left(p_{j}-p_{j+1}\right)\left(\rho_{j}-\rho_{j+1}\right)\right] .
\end{array}
$$

Furthermore, the last term can be omitted to guarantee that $\mathcal{D}$ be positive,

$$
\mathcal{D}=\frac{1}{\rho_{j} \rho_{j+1}}\left[M_{*}^{2}\left(p_{j}+p_{j+1}\right)\left(\rho_{j}+\rho_{j+1}\right)\right],
$$

which looks similar to the first expression. The scheme with the inclusion of the pressure diffusion term $(\mathrm{Eq} .(22))$ is now denoted as $\mathrm{AUSM}^{+}$. ap.

We now make remarks on the preconditioning matrix $\Gamma$. We have used the Weiss-Smith $\Gamma[18]$ to arrive at the scaling function $f_{a}\left(\bar{M} ; M_{*}\right)$ in Eq. (12). Other preconditioners [12-14] can be used as well. The procedure for extension will be precisely the same since all one needs is the eigenvalues of the preconditioned hyperbolic system. Thus, a new numerical speed of sound $\tilde{a}$ can be expressed in terms of the scaling function $f_{a}\left(\bar{M} ; M_{*}\right)$. However, no significant effect on the solution is anticipated because all these preconditioners yield more or less the same behavior in the limits of $M \rightarrow 0$ and 1 . Unless at low speed (say $M_{\infty}<0.3$ ), it was found in our calculations not necessary to include the preconditioning matrix in solving the governing equations. In other words, the scaling function can be incorporated alone, as in Eqs. (12)-(22), in the numerical flux and improvements in accuracy and convergence can be realized.

\section{RESULTS AND DISCUSSION}

In this section, we will present $2 \mathrm{D}$ and $3 \mathrm{D}$ Navier-Stokes solutions for turbulent flows over various geometries. The scheme proposed in this paper was implemented in the OVERFLOW code that has been developed by Buning et al. [6]. The LHS operator was approximated with the standard central difference scheme plus appropriate artificial damping terms, (even though the RHS residual operator was represented with an upwind scheme!), it was then further factored and diagonalized in each space dimension. To accelerate convergence, a full multigrid strategy using two levels of coarser grids is applied initially.

The flux in the RHS operator was constructed with a third-order accurate interpolation for the primitive variables, together with limiter used by Koren [19]. The cutoff Mach number in Eq. (15) is given by $M_{c o}^{2}=M_{\infty}^{2} / 4$. Also, the OVERFLOW code has a parameter controlling the use of the preconditioning matrix $\boldsymbol{\Gamma}$. We kept the default value to be $3 M_{\infty}^{2} \leq 1$ under which condition $\boldsymbol{\Gamma}$ was activated.

For all calculations presented in this paper, the flow is assumed fully turbulent no laminar or transition regions are consider. Unless stated otherwise, the baseline model is the SpalartAllmaras one-equation model. But the effects of various turbulence models on the solutions in comparison with measured data will also be studied.

In this paper we will demonstrate the effectiveness of using the numerical speed of sound in 
calculating flows at all speeds, specifically focusing on two issues: (1) convergence rate and (2) accuracy.

\section{NACA Airfoil}

A C-grid of $249 \times 56$ points was used for the NACA 0012 airfoil. The purpose of calculating this flow was to determine the performance of five popular turbulence models against the experimental data, see [20], so that it may help down-select fewer models for additional validation tests. These five models include the Baldwin-Lomax (denoted as BL) [21], BaldwinBarth (BB) [22], Spalart-Allmaras (SA) [23], Mentor k- $\epsilon$ (SST) [24], and $k-\omega$ [25] models. Figure 2 displays the surface pressure coefficient on the airfoil, indicating a relatively strong shock situating in the middle of the suction surface of the airfoil. In fact the boundary layer is separated because of the adverse pressure set up by the shock. The Baldwin-Lomax model underestimates the effects of shock/boundary layer interaction, resulting in an incorrect shock location; but it gives the best result on the pressure side. The Baldwin-Barth one-equation model gives a very good prediction of the shock location, but with some disagreement with the data on the pressure side. The Spalart-Allmaras oneequation model overall gives a good agreement with the data. The suction-side pressure is best predicted by the SST model, but the pressure side is accurately predicted by the $\mathrm{BL}$ and $\mathrm{k}$ $\omega$ models. Figure 3 displays the pressure contours resulting from all five turbulence models. Noticing that the models that predict the shock location correctly appear to have a shock profile curving forward more pronouncedly at the foot. This behavior also corresponds to the fact of having a larger separated bubble.

The calculations were carried out for all models using the same set of parameters associated with time marching procedures and the implicit operator, which are by no means the optimal set for any models. The convergence histories of these five models are shown in Fig. 4, where the SA model reaches the steady value of $C_{L}$ in about 2,000 time steps, while the $\mathrm{BL}$ model takes about
3,700 steps. It is interesting that the lift coeffcients from the $\mathrm{BL}$ and $\mathrm{BB}$ models starts out at a low value and increases to an asymptote as iteration proceeds and a completely opposite trend is observed for the other models.

\section{Shuttle External Tank}

This is an axisymmetric Shuttle external tank geometry with a sharp nose and blunt base, downstream of which there is a significant separation zone. One of the grid lines conforms to the body and grows outward and a plane consists of $88 \times 60$ grid points. Shown also are the meshes clustered around some key regions, one of which is in the middle to resolve a tiny notch (not visible to the scale). The free stream Reynolds number was fixed at 10,000 . We have tested conditions from low Mach, transonic, to supersonic flows. Several schemes were considered, consisting of the standard $\mathrm{AUSM}^{+}, \mathrm{AUSM}^{+}-\mathrm{a}$, and $\mathrm{AUSM}^{+}$ap, with and without the Weiss-Smith preconditioner. In all calculations for this problem, we made 200 steps for each of two coarser grids prior to the finest grid, on which 3000 more steps were continued unless noted otherwise.

Table I summarizes the convergence behavior of the above combinations. We observe the following: (1) For low Mach numbers (approximately $M_{\infty}<0.3$ ), it was found necessary to use the time-derivative preconditioner $\Gamma$ so that the numerical dissipations in both the implicit and explicit operators are compatibly scaled. Otherwise, the calculation either diverged or stagnated. (2) For flows at transonic speeds or higher, the time-derivative preconditioner, as given in Eq. (4), serves no benefits whatsoever, even though the fluid speed is low in the viscous and the base flow regions. This is understandable because the preconditioner effects only the inviscid waves and the information in the viscous-dominated regions is only transmitted via diffusion processes which are ably handled by the implicit operator.

In Fig. 5, we display the convergence history for various Mach numbers using $\mathrm{ACSM}^{+}$, but without the preconditioner $\Gamma$. The residuals for 
the low Mach-number cases stall after a drop of four orders of magnitude. These drops in many calculations, although not especially admirable, would have been acceptable. However, a close examination of the solution reveals that it is completely unacceptable, as shown in Fig. 6. It appears that there is a false boundary (exactly aligned with a grid line) at which information is unable to get passed. This phenomenon is quite typical in the low Mach-number calculations using an unmodified compressible code, also seen in [5]. Hence, a measure of caution should be taken when reading the residual history for the low Mach-number solutions.

On the other hand, the convergence histories with the use of the numerical speed of sound display improvement over those without it, as shown in Fig. 5. As noted earlier in Table I, it is necessary to invoke $\boldsymbol{\Gamma}$ for the $M_{\infty}=0.01$ and 0.1 cases. The convergence rates for these two calculations nearly coincide with each other, indicating Mach-number independence.

In Fig. 7 , we show the solution at $\mathrm{N}=1000$ steps at which the residual has been dropped to the level approximately equal to that of the baseline scheme $\mathrm{AUSM}^{+}$, at $\mathrm{N}=6400$. However, the solution now is well behaved and is every bit as good as the final solution at $\mathrm{N}=3200$ at which the residual has been further reduced by two orders. Also the blow-up view near the surface reveals smooth profiles of pressure contours, unlike the standard $\mathrm{AUSM}^{+}$which has been known to yield unwanted pressure oscillations in viscous layers along the transverse grid lines when the mesh aspect ratio is large and flow is essentially parallel to a grid line.*

Finally the effect of including the pressure diffusion term on the solution was investigated and the convergence histories are also included in Fig. 5. Again, the preconditioner $\Gamma$ must be used for the low Mach-number cases and their convergence histories are essentially identical, becoming independent of Mach number as the Mach number lowers. The pressure contours are indistinguishable from those shown in Fig. 7 and are thus not included.
Comparing results in Fig. 5, we see that the convergence rate is improved in the transonic ranges by simply using the numerical speed of sound alone. For low Mach number cases, $M_{\infty}=$ $0.01,0.1$, another order of reduction can be obtained by including the pressure diffusion term. Also, the use of numerical speed of sound yields the convergence histories that are relatively insensitive to the flow speeds.

We now summarize major findings from the study of this problem: (1) The numerical speed of sound concept is an effective means of extending the AUSM-type discretization to solve low Mach number flows in an accurate and efficient manner. (2) Since the numerical speed of sound is reduced with the flow speed, the numerical dissipation changes accordingly, and a compatible implicit operator (one that includes the preconditioning matrix) must be used. (3) For moderate Mach numbers and beyond, it is not necessary to use $\boldsymbol{\Gamma}$, (4) Incorporation of the numerical speed of sound, as described in steps (12)-(22)), helps remove pressure oscillations in the viscous layers.

\section{ONERA M6 Wing}

The next problem is the ONERA M6 wing with the free stream conditions $M_{\infty}=0.84$, and $R e_{\infty}=18.2 \times 10^{6}$ under various angles of at tack. The computation domain consists of $269 \times 35 \times 67$ grid points. For this case, the preconditioning matrix $\Gamma$ was automatically turned off in the code since the controlling factor $3 M_{\infty}^{2}$ exceeds unity. However, the numerical speed of sound $\tilde{a}$ was active with $M_{c o}=M_{x} / 2$. The pressure contours on the wing surfaces are displayed in Fig. 8, showing the well-known $\lambda$ shock pattern, where the double-shock region extends more than $80 \%$ of the wing span. The detailed comparison of surface pressure distributions are shown for three spanwise sections, Figs. 9,10 and 11 respectively for $44 \%, 65 \%$, and $90 \%$, for three angles of attack. The computed results are in very good agreement with the data [26],

*However, the pressure distribution along the wall is smooth. 
noticing the accurate capturing of the shock locations. Recall that the SA model is assumed, unless noted otherwise.

The convergence histories are presented in Fig. 12 for three different angles of attack. They show a continuing decrease in 800 steps (600 fine-grid steps) by about four orders of magnitude from the largest values, at nearly the same rates even though the flow features have varied considerably.

Figure 13 compares the effect of three turbulence models, SA, $i-\omega$, and SST models, on the pressure prediction; the SA and SST models give nearly identical results, except minor differences behind the shock at $\eta=0.95$, and the $\kappa-\omega$ model seems to give a slightly better agreement with the data for the shock location. The particle traces of two angles of attack, $\alpha=3.06$ and 5.06, at different wingspans are given in Fig. 14, showing little spanwise excursion, except at the wing tip for the $\alpha=3.06^{\circ}$ case, but appreciable three-dimensional effect for the other case.

\section{Wingbody}

Turbulent flows over a wingbody configuration were calculated. The geometry is shown in Fig. 15 , where the sting is included in the calculation. The computation domain was gridded using the chimera overset grid technique and entire grid composed of seven grids. The particle traces around the body and wing, shown in Fig. 16, indicate that the flow follows the configuration quite closely and is essentially two-dimensional over the wing. Figure 17 depicts the pressure coefficients at various spanwise locations. The computed results are in excellent agreement with the measured data [27]. Moreover, the pressure coefficients along the body, shown in Fig. 18 , exhibits the similar level of excellent agreement with the data. Even in the wing root region where a sharp variation is encountered.

Finally, Fig. 19 displays a well-behaved convergence history, reducing the residual error by more than five (5) orders of magnitude in 800 steps.

\section{CONCLUDING REMARKS}

We have successfully implemented the new $\mathrm{AUSM}^{+}$flux scheme in the production code of NASA, OVERFLOW and presented in this paper the validation results of test cases. With the introduction of the numerical speed of sound in the previous $\mathrm{AUSM}^{+}$flux scheme, it now yields accurate results at low Mach number and efficient convergence at a rate (nearly) independent of Mach number and angle of attack. Interestingly, the convergence rate is even enhanced at transonic speeds with the numerical speed of sound, without invoking the preconditioning matrix. Effect of turbulence models on the results has also been investigated, the SA and SST models give the best results in comparison with the measure data of NACA 0012 airfoil and ONERA M6 wing.

\section{ACKNOWLEDGMENTS}

This task was supported by the Turbomachinery and Combustion Technology and High Performance Computing and Communication Projects, through the Subsonic Systems Office and Computing and Interdisciplinary Systems Office respectively, Glenn Research Center.

\section{REFERENCES}

1. M.-S. Liou and C. J. Steffen, J. Comput. Phys. 107, 23 (1993).

2. M.-S. Liou, NASA TM 106524 (1994); also $J$. Comput. Phys. 129, 364 (1996).

3. Y. Wada and M.-S. Liou, SIAM J.Sci. Comput. 18,633 (1997).

4. M.-S. Liou, AIAA Paper 97-2035-CP, 1997

5. M.-S. Liou and J. R. Edwards, AIAA Paper 99-3268-CP, 1999.

6. P. G. Buning et. al., "OVERFLOW User's Manual, version 1.8f" Unpublished NASA Report, 1998 .

7. R. Radiespiel J. Comput. Phys 121 (1995).

8. R. Takaki, et al. First Europe-US High Speed Flow Field Database Workshop II, 1997. 
9. D. Darracq, S. Champagneux, and A. Corjon, AIAA Paper 98-2411, 1998.

10. J. H. Lee and O. H. Rho, AIAA Paper 98$1538,1998$.

11. J. R. Edwards and M.-S. Liou, AIAA J. 36, 1610 (1998).

12. Y. H. Choi and C. L. Merkle, J. Comput. Phys. 105, 207 (1993).

13. E. Turkel, V. N. Vatsa and R. Radespiel, AIAA Paper 96-2460, 1996.

14. B. van Leer, W. T. Lee and P. L. Roe, AIAA paper $91-15.52,1991$.

15. R. Beam and R. Warming, ALAA J. 16, 393 (1978).

16. T. H. Pulliam and D. S. Chaussee, J. Comput. Phys. 39, 347 (1981).

17. J. R. Edwards, Franklin and M.-S. Liou, AIAA 99-3327-CP, 1999.
18. J. M. Weiss and W. A. Smith, AIAA Paper 94-2209, 1994.

19. B. Koren, Lecture Notes in Physics 323, 344 (1989).

20. T. L. Holst, AIAA J. Aircraft 25, 1073 (1988).

21. B. S. Baldwin and H. Lomax, AIAA Paper $78-0257,1978$.

22. B. S. Baldwin and T. J. Barth, AIAA Paper 91-0610, 1991.

23. P. R. Spalart and S. R. Allmaras, AIAA Paper 92-0439, 1992.

24. F, R. Mentor, AIAA J. 32, 1598 (1994).

25. D. C. Wilcox, AIAA J. 26, 1299 (1988).

26. V. Schmitt and F. Charpin, Appendix B1, AGARD-AR-138, 1984.

27. D.A. Treadgold, A.F. Jones, and K.H. Wilson, Appendix B4, AGARD-AR-138, 1984.

Table 1: Summary of convergence behavior due to various schemes for the shuttle external tank, $R \epsilon_{\infty}=10000$.

\begin{tabular}{|c|c|c|c|}
\hline$M_{\infty}$ & Scheme & No Precond. & Precond. \\
\hline \hline \multirow{3}{*}{0.01} & AUSM $^{+}$ & $\sqrt{ }$ & Diverg. \\
& AUSM $^{+}-\mathrm{a}$ & No converg. & $\sqrt{ }$ \\
& AUSM $^{+}-\mathrm{a}+\dot{m}_{p}$ & No converg. & $\sqrt{ }$ \\
\hline \hline \multirow{3}{*}{0.80} & AUSM $^{+}$ & $\sqrt{ }$ & $\sqrt{ }$ \\
& AUSM $^{+}-\mathrm{a}$ & $\sqrt{ }$ & $\sqrt{ }$ \\
& $\mathrm{AUSM}^{+}-\mathrm{a}+\dot{m}_{p}$ & $\sqrt{ }$ & $\sqrt{ }$ \\
\hline \hline \multirow{2}{*}{2.00} & $\mathrm{AUSM}^{+}$ & $\sqrt{ }$ & $\sqrt{ }$ \\
& $\mathrm{AUSM}^{+}-\mathrm{a}$ & $\sqrt{ }$ & $\sqrt{ }$ \\
& AUSM $^{+}-\mathrm{a}+\dot{m}_{p}$ & $\sqrt{ }$ & $\sqrt{ }$ \\
\hline
\end{tabular}



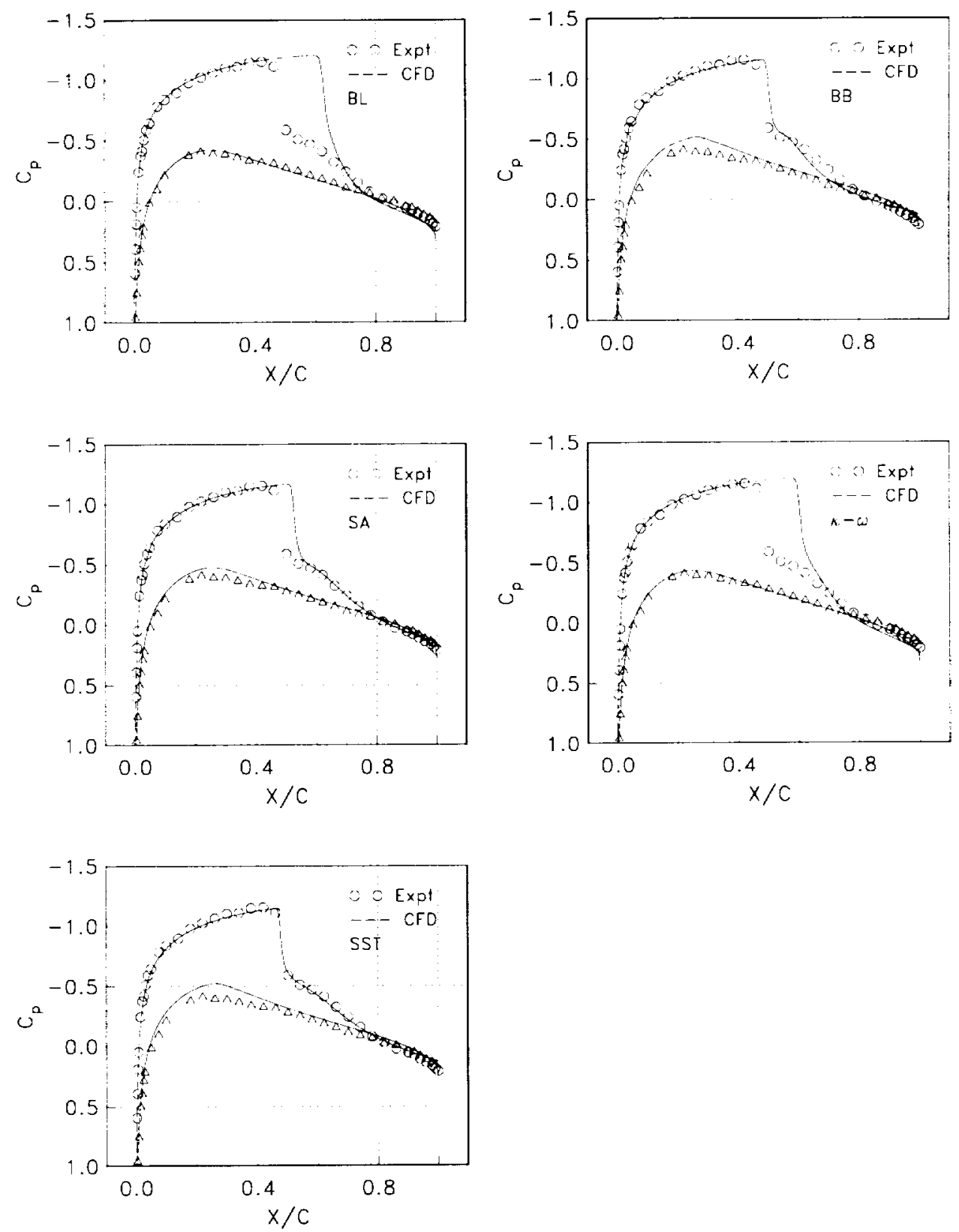

Fig. 2 Pressure coefficients on the NACA 0012 airfoil, using various turbulence models: $M_{\infty}=0.799, \alpha=2.26^{\circ}$. 

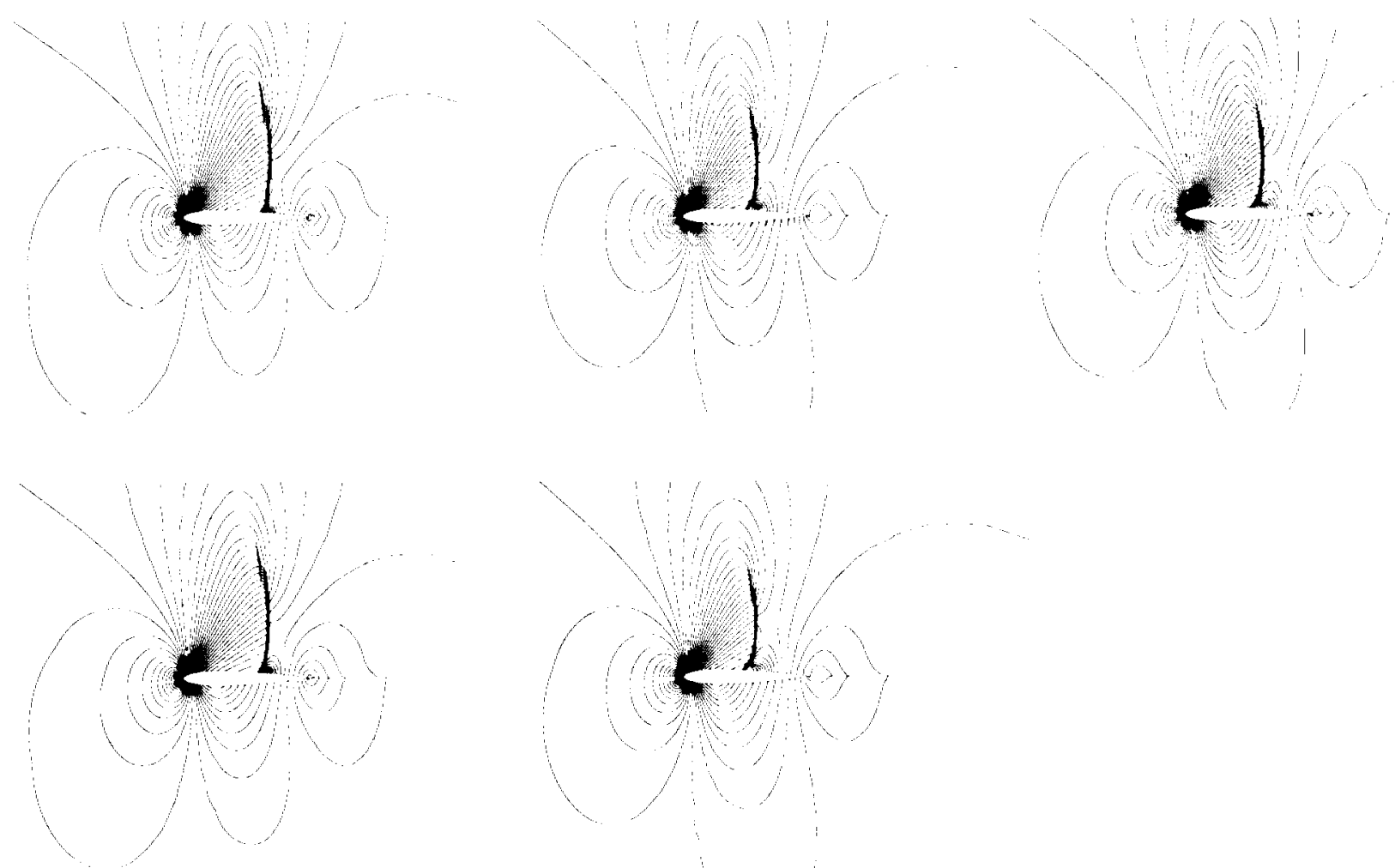

Fig. 3 Pressure contours on the NACA 0012 airfoil predicted by various models. From left to right, Top row: BL, BB, and SA; Bottom row: k-j and SST.
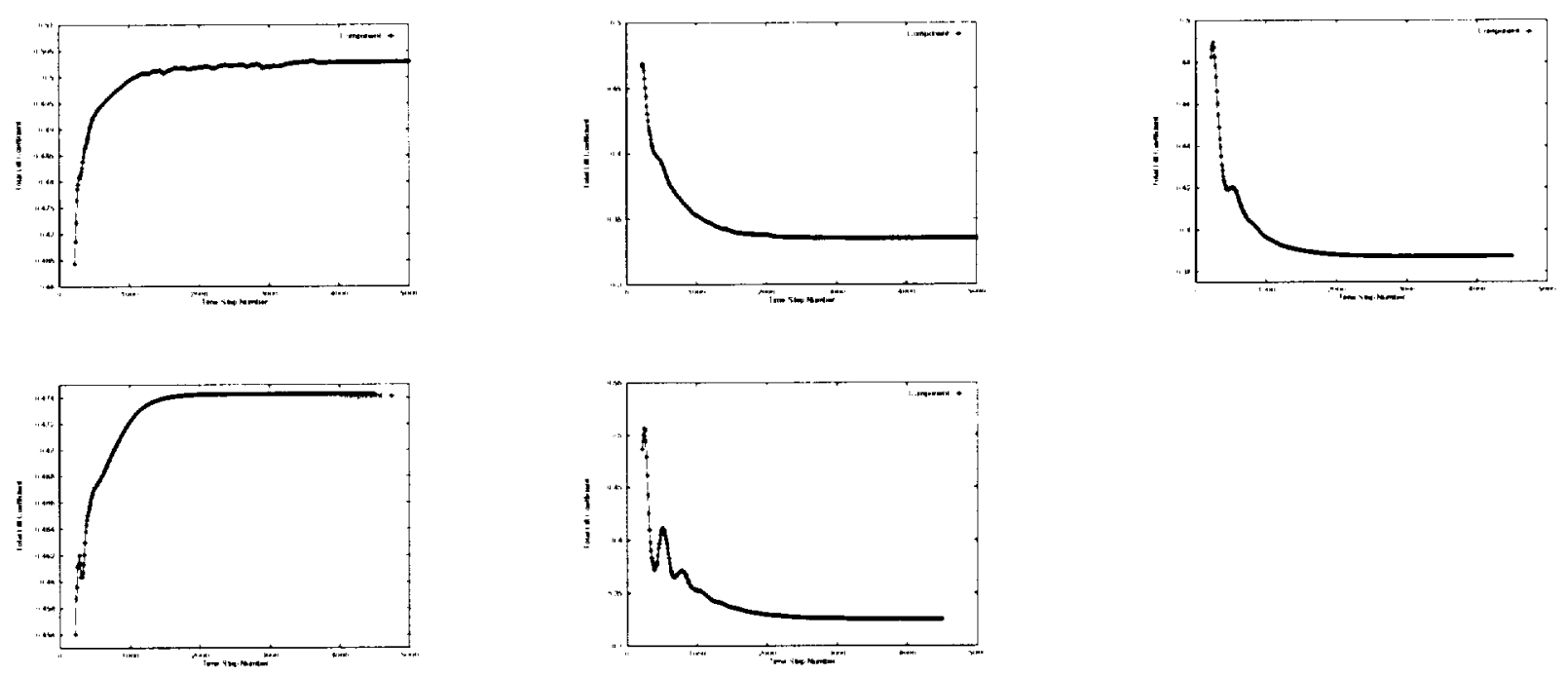

Fig. 4 Comparison of convergence histories in $C_{L}$ among different turbulence models. From left to right, Top row: $\mathrm{BL}, \mathrm{BB}$, and $\mathrm{SA}$; Bottom row: $k-\omega$ and $\mathrm{SST}$. 

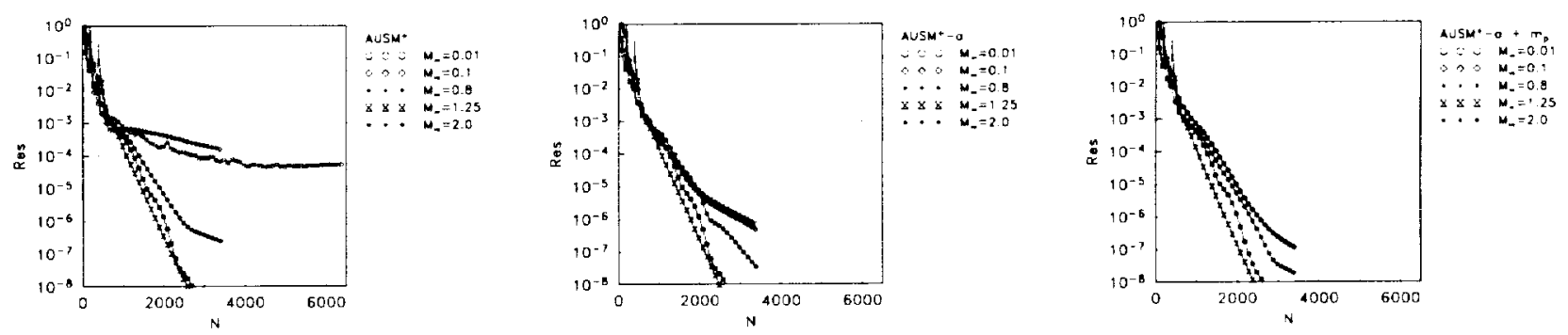

Fig. 5 Convergence history for the shuttle external tank problem: (Left) ALSM ${ }^{+}$, (Middle) ALSM ${ }^{+}$a, (Right) $\mathrm{AUSM}^{+}-$ap.
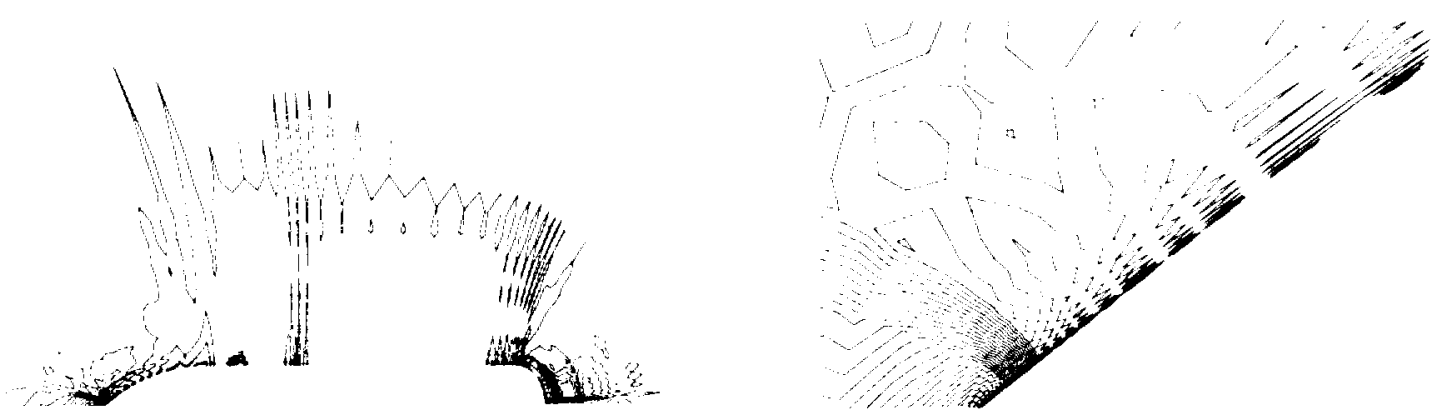

Fig. 6 Pressure contours for the shuttle external tank problem obtained at $\mathrm{N}=6400$ time steps for $M_{\infty}=0.01$, using the standard AUSM+. The picture to the right shows a magnified view near the nose.
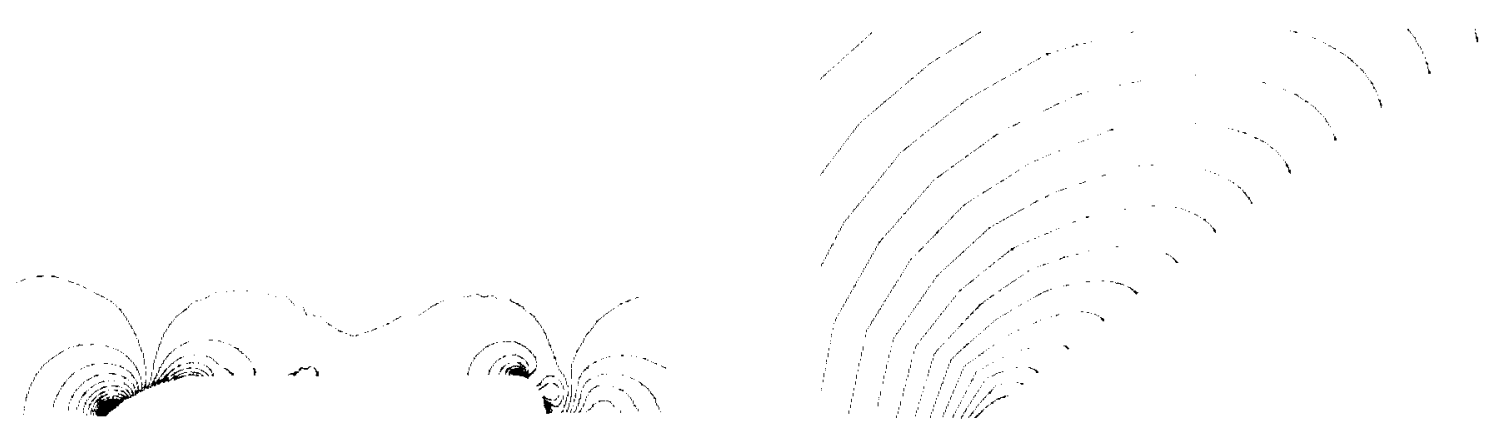

Fig. 7 Pressure contours for the shuttle external tank problem obtained at $\mathrm{N}=1000$ time steps for $M_{\infty}=0.01$, using $\mathrm{ALSM}^{+}-\mathrm{a}$. The other picture shows a magnified view near the nose. 

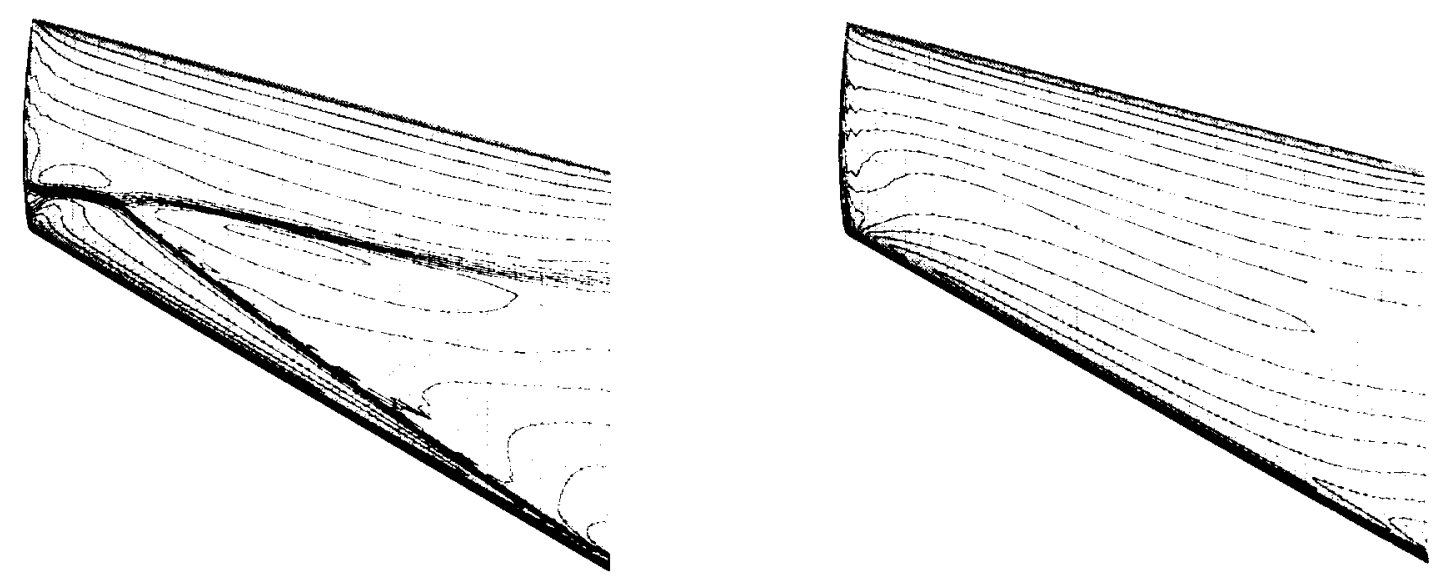

Fig. 8 Pressure contours on the pressure and suction surfaces of the ONERA M6 wing at $\alpha=3.06^{\circ}, M_{\infty}=$ 0.84 , and $R e_{\infty}=18.2 \times 10^{6}$, showing the $\lambda$-shock pattern near the wing tip.
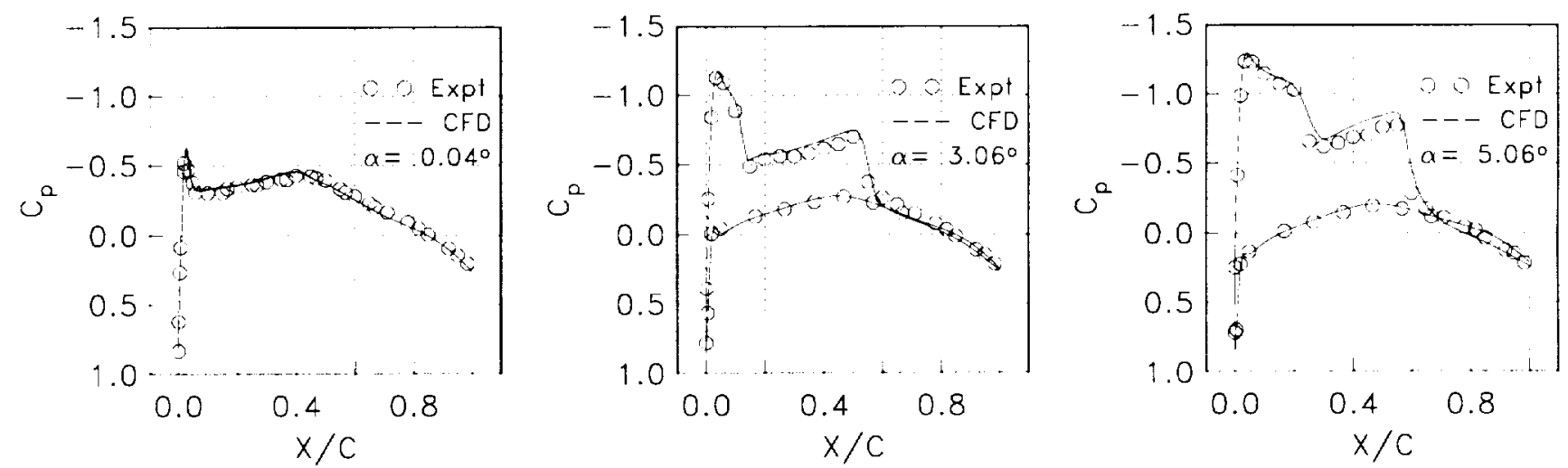

Fig. 9 Pressure distribution at the spanwise section, $44 \%$ of the ONERA M6 wing, for various angles of attack, $M_{\infty}=0.84, R e_{\infty}=18.2 \times 10^{6}$.
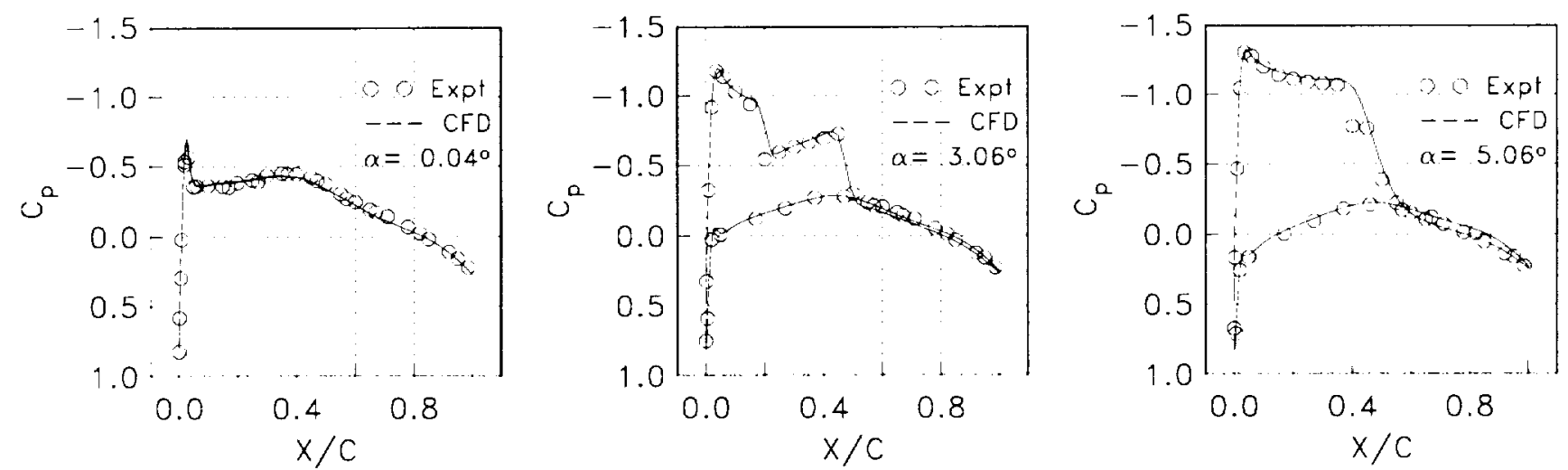

Fig. 10 Pressure distribution at the spanwise section, $65 \%$ of the ONERA M6 wing, for various angles of attack, $M_{\infty}=0.84, R e_{\infty}=18.2 \times 10^{6}$. 

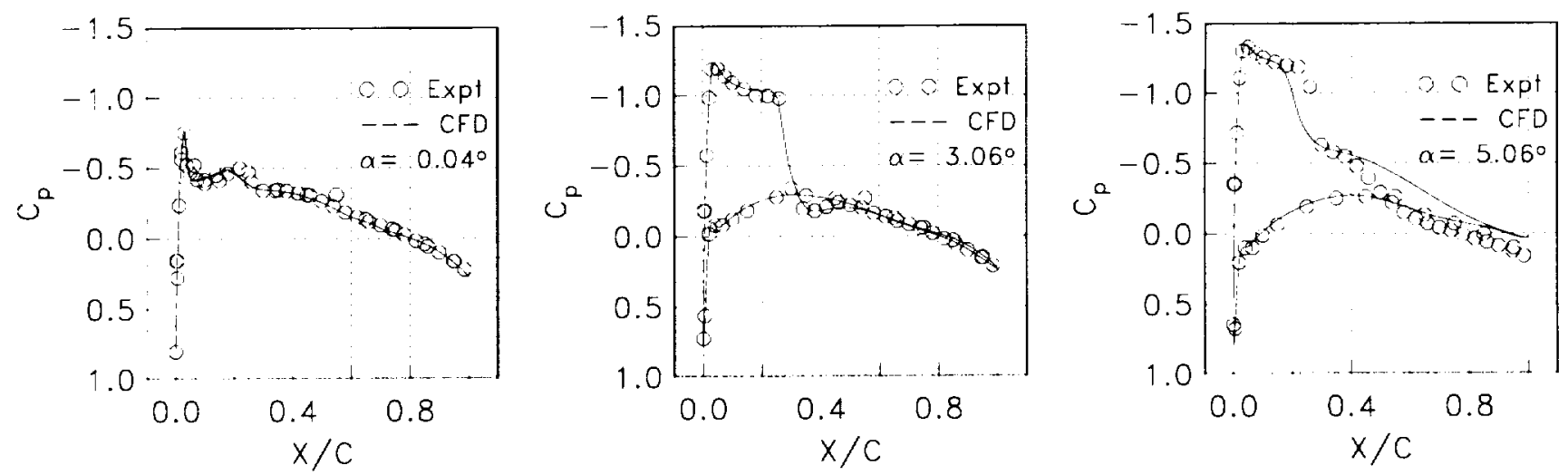

Fig. 11 Pressure distribution at the spanwise section, 90\% of the ONERA M6 wing, for various angles of attack, $M_{\infty}=0.84, \operatorname{Re}_{\infty}=18.2 \times 10^{6}$.

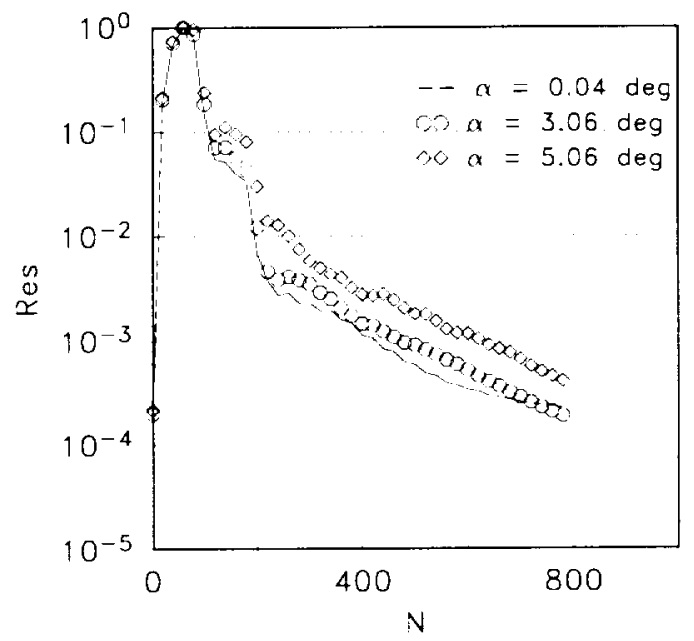

Fig. 12 Convergence history for the ONERA M6 wing problem at three angles of attack, $M_{\infty}=0.84, \operatorname{Re} e_{\infty}=$ $18.2 \times 10^{6}$.
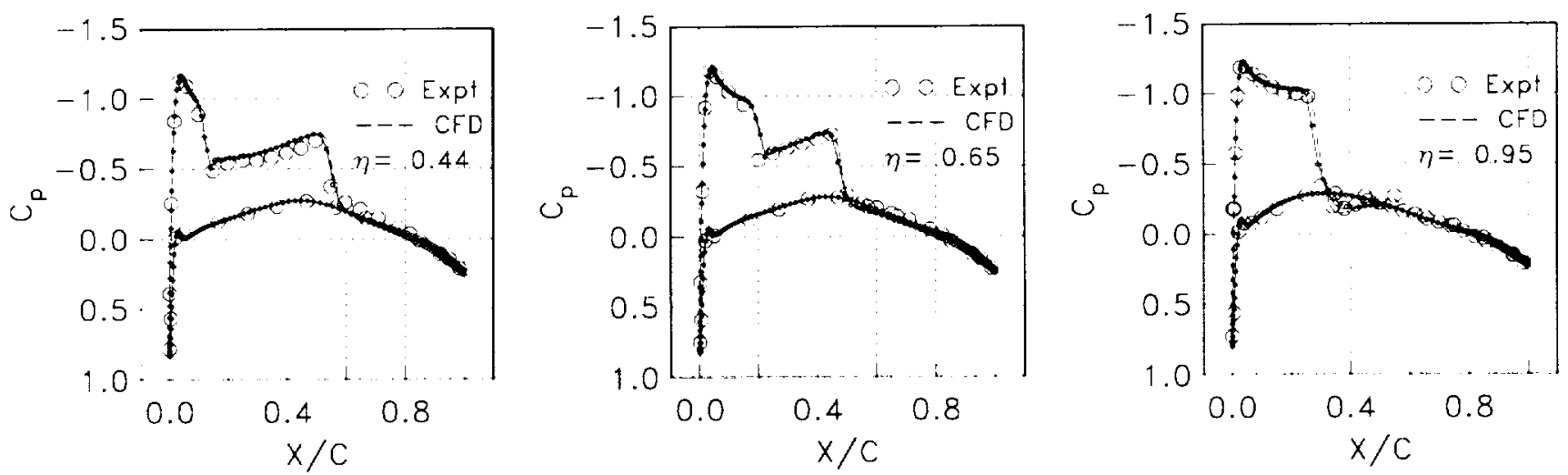

Fig. 13 Effect of turbulence models on the prediction of pressure distributions for the ONERA M6 wing problem at $\alpha=3.06^{\circ}, M_{\infty}=0.84, R e_{\infty}=18.2 \times 10^{6}$.; Solid line: SA model, dash line: $\kappa-\omega$ model, and small dot: SST model. The SA and SST results are nearly identical, except some differences behind the shock at $\eta=0.95$. 

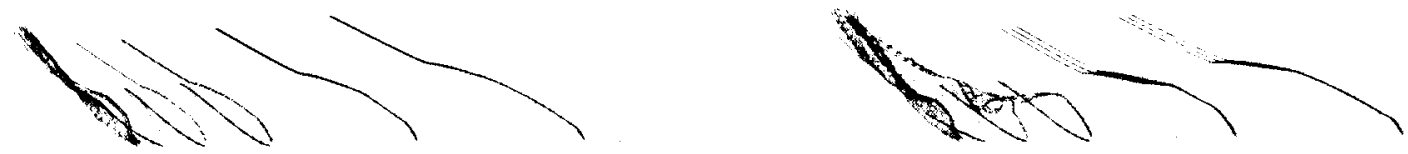

Fig. 14 Particle traces for the ONERA M6 wing problem at $M_{\infty}=0.84, R e_{\infty}=18.2 \times 10^{6}$., and Left: $\alpha=3.06^{\circ}$, Right: $\alpha=5.06^{\circ}$.

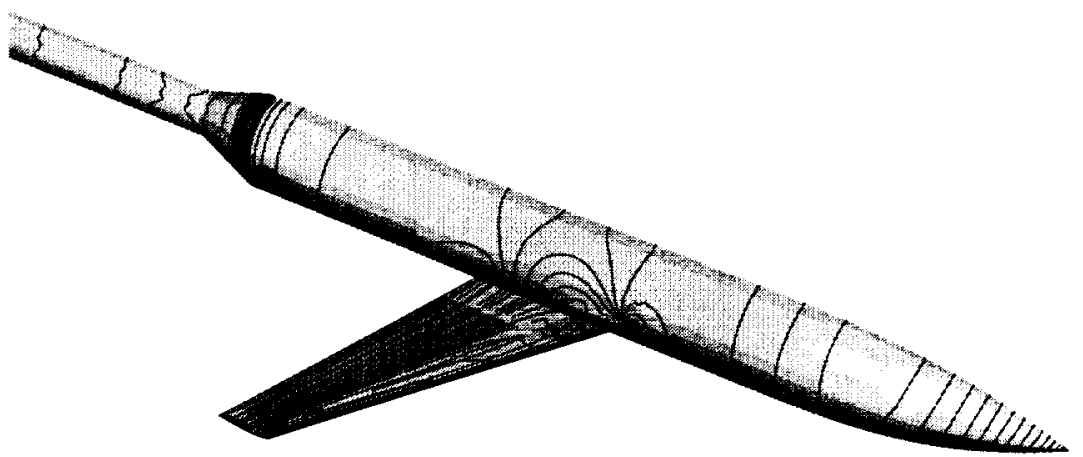

Fig. 15 Geometry of the wingbody problem.

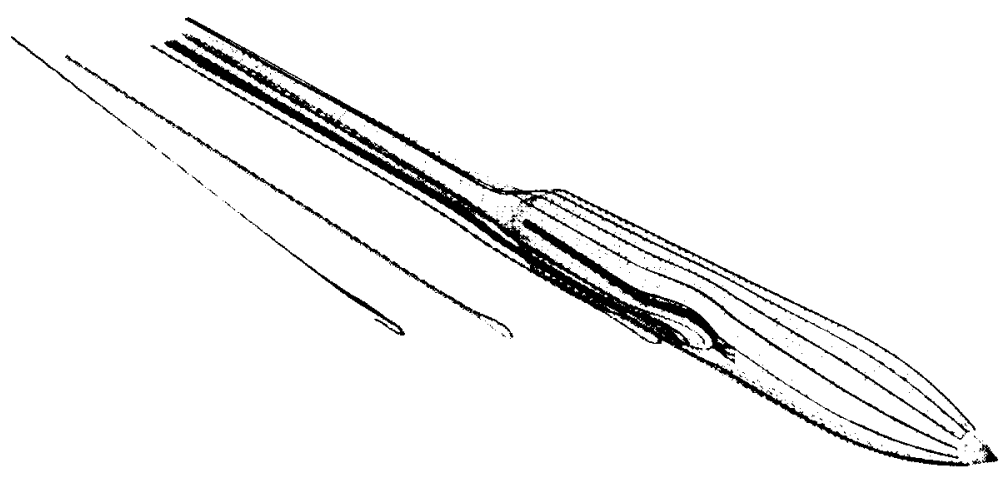

Fig. 16 Particle traces around the body and wing. 

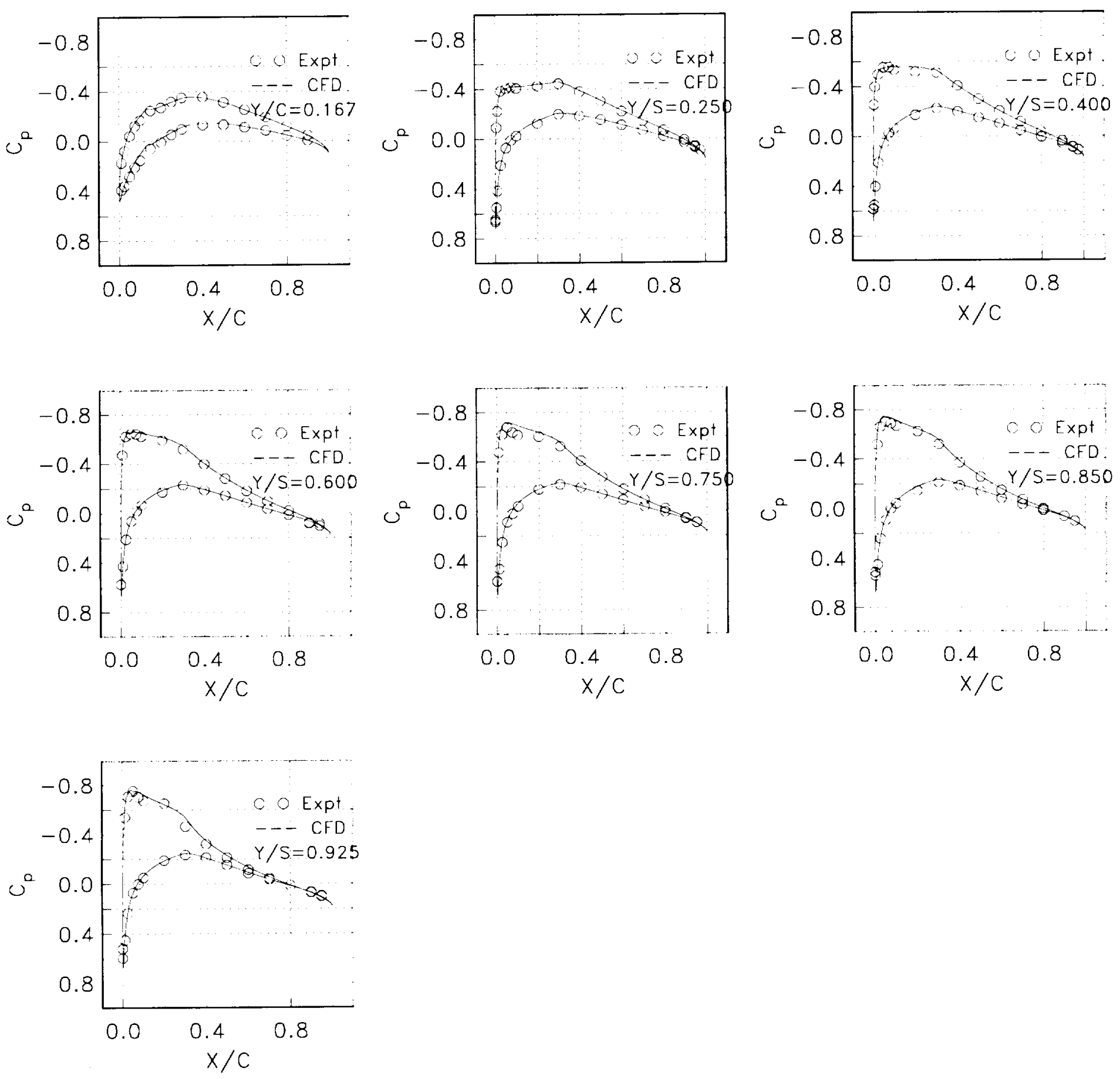

Fig. 17 Pressure distribution at various sections of the wing, $M_{\infty}=0.8, \alpha=2^{\circ} . R e_{\infty}=0.167 \times 10^{6}$. 

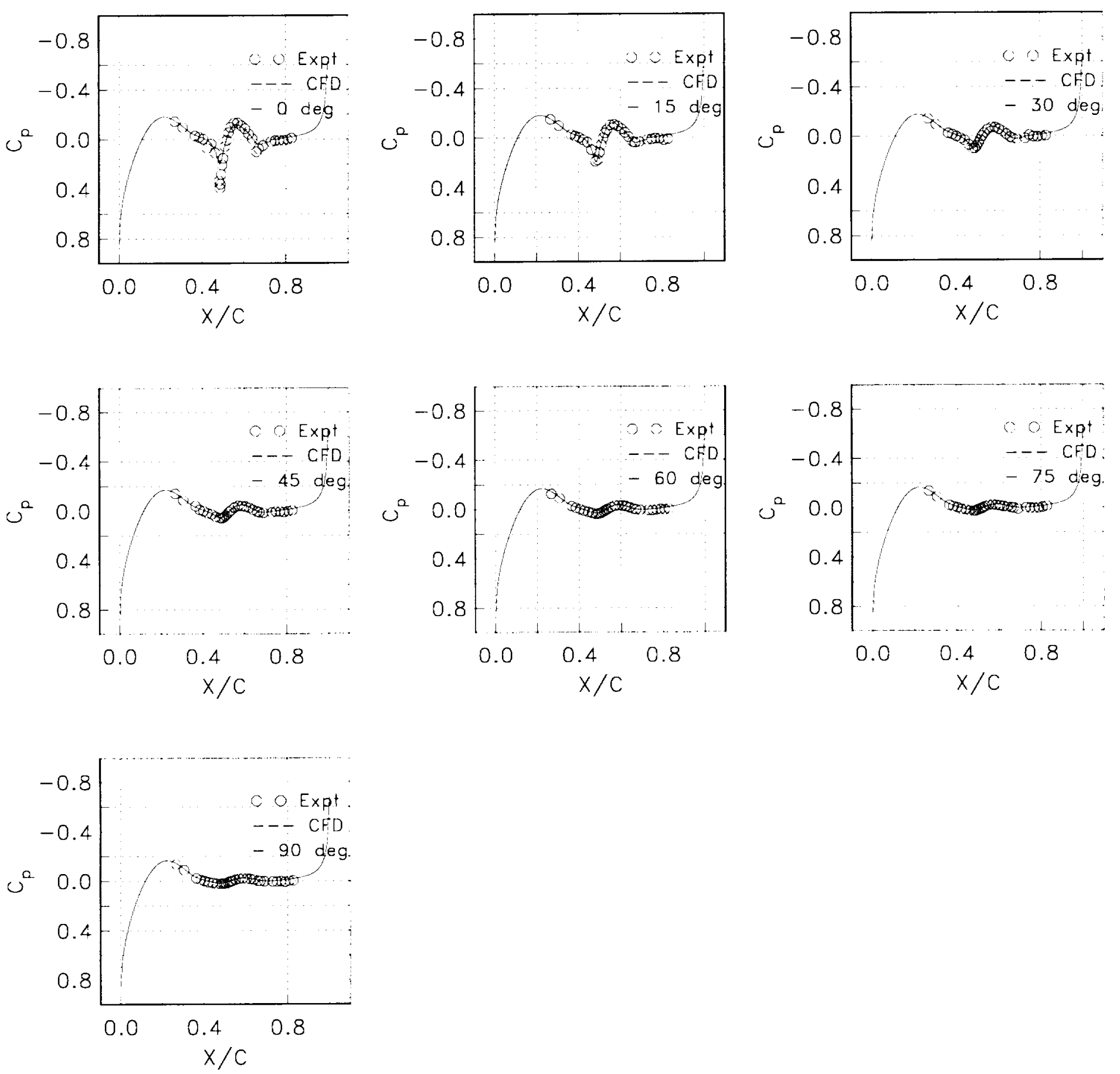

Fig. 18 Pressure distribution at angles along the body, $M_{\infty}=0.8, \alpha=2^{\circ}, R e_{\infty}=0.16 \mathrm{\jmath} \times 10^{6}$. 


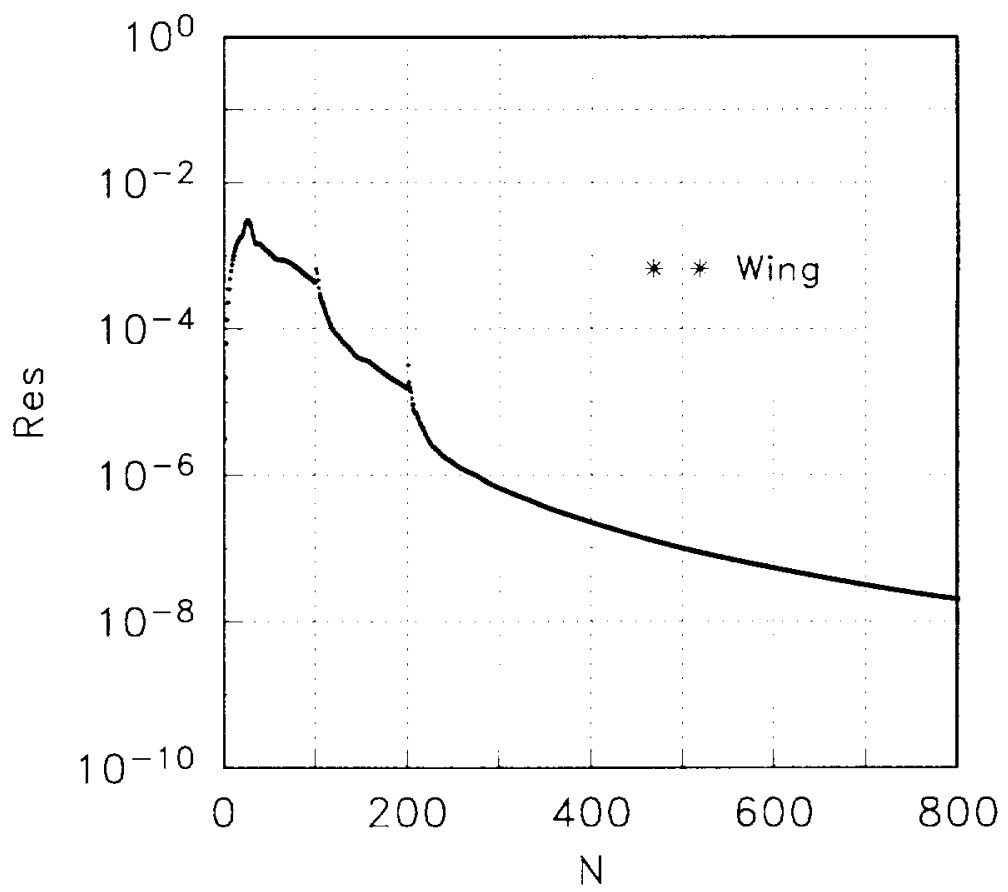

Fig. 19 Convergence history for the wingbody problem. 